\title{
Erratum
}

\section{Erratum to: Asymptotic estimates and stability analysis of Kuramoto-Sivashinsky type models}

Milena Stanislavova And Atanas Stefanov

\section{Erratum to: J. Evol. Equ.}

\section{DOI 10.1007/s00028-011-0103-5}

In our paper, there was an erroneous claim that all the stationary solutions of the Kuramoto-Sivashinsky equation,

$$
u_{t}+u_{x x x x}+u_{x x}+u u_{x}=0, \quad-L \leq x \leq L
$$

are spectrally and linearly unstable. That is, we have claimed that the linearized operator

$$
\mathcal{L} z=z_{x x x x}+z_{x x}+\partial_{x}(\varphi z)
$$

necessarily has an unstable eigenvalue (that is an eigenvalue with negative real-part). In our justification for this claim, we have correctly verified a necessary condition, which is however not a sufficient one. In fact, it turned out that there are stable as well as unstable stationary solutions to (1), see for example [1].

Thus, the statement of linear instability (namely part 2) in Proposition 2 is invalid. We regret this error, and we are grateful to our colleagues M. Johnson and K. Zumbrun for bringing this to our attention.

Another issue that came up during our discussions with Johnson and Zumbrun is the implausibility of the spectral condition $(A)$ that we have imposed on the operator $\mathcal{L}$. Namely, we have required that

$$
\sigma(\mathcal{L}) \cap\{\Re z=0\}=\{0\} \text { and } 0 \text { is a simple eigenvalue. }
$$

\footnotetext{
The online version of the original article can be found under doi:10.1007/s00028-011-0103-5.
} 
The reasoning behind this requirement was that there is only translational invariance, associated with (1) and thus 0 should be "physically" of dimension one (with eigenfunction $\varphi^{\prime}$ ). As it turned out, there is another symmetry, that was left unaccounted for, namely $c+u(t, x-c t)$ is a solution, whenever $u$ is one. This symmetry makes the zero of geometric multiplicity at least two, since $\mathcal{L}(1)=\varphi^{\prime}$. Thus, a physically reasonable assumption would be the following

$$
\sigma(\mathcal{L}) \cap\{\Re z=0\}=\{0\} \text { and } 0 \text { generates a Jordan block of length two. }
$$

We note that even $B$ is not always satisfied, per the numerical computations of the spectra of $\mathcal{L}$ in [2].

Miraculously, our main result (Theorem 4) about conditional asymptotic stability of such stationary solution survives this unfortunate adjustments. In fact, we can in addition say that spectrally stable stationary solutions of (1) are in fact asymptotically stable. Here is the precise formulation of the main result.

THEOREM 1. Assume $\varphi$ is a time-independent solution of (1), and let ${ }^{1}$ $N:=\operatorname{dim}\left[\operatorname{Ran}\left(P_{<0}(\mathcal{L})\right)\right]$. Assume also that the linearization $\mathcal{L}$ satisfies the spectral hypothesis $(B)$. Then, $\varphi$ is asymptotically stable, if $N=0$ and conditionally asymptotically stable if $N \geq 1$.

More precisely, there exists a co-dimension $N$ manifold $\mathcal{M}_{s} \subset H_{\text {per }}^{1}[-L, L]$ and $\varepsilon=\varepsilon(L, \varphi)>0$, so that whenever $u_{0}-\varphi \in \mathcal{M}_{s}$ is real-valued and $\left\|u_{0}-\varphi\right\|_{H_{p e r}^{1}[-L, L]}<\varepsilon$, there exists $y^{\infty}=y^{\infty}\left(u_{0}\right) \in \mathbf{R}$, so that for $c_{0}=(2 L)^{-1} \int_{-L}^{L}\left(u_{0}(x)-\varphi(x) \mathrm{d} x\right.$,

$$
\begin{aligned}
& u(t, x)=c_{0}+\varphi\left(x+y(t)-c_{0} t\right)+v\left(t, x-c_{0} t\right), \\
& y(t)=\left(1-\mathrm{e}^{-A t}\right) y^{\infty} \\
& \sup _{t \geq 0} \mathrm{e}^{\delta t}\|v(t, \cdot)\|_{H_{p e r}^{1}} \leq 2 \varepsilon .
\end{aligned}
$$

where $A$ is sufficiently large real, for some $\delta=\delta(\mathcal{L})>0$.

As one can see, if $\int_{-L}^{L} u_{0}(x)=\int_{-L}^{L} \varphi(x) \mathrm{d} x$, it follows that $c_{0}=0$, and the statement does not differ from our original statement. This is a consequence of the fact that in this case, the evolution proceeds trivially on the "forgotten" adjoint eigen-direction $\psi=$ const. $=1$. We quickly indicate how the proof of Theorem 1 reduces to the original proof of Theorem 4 .

Take the ansatz $u(t, x)=c_{0}+\varphi\left(x+y(t)-c_{0} t\right)+v\left(t, x-c_{0} t\right)$, where $y(t)=$ $\left(1-\mathrm{e}^{-A t}\right) y^{\infty}$ and $y^{\infty}, c_{0}$ are parameters to be determined (nonlinearly) from initial data. By the mean preservation property $\int_{L}^{L} u(t, x)=$ const., and periodicity, we

\footnotetext{
${ }^{1}$ Here if $N=0$, we have a linearly stable solution, whereas, if $N \geq 1$, we have linearly unstable solution.
} 
conclude

$$
2 L c_{0}+\int_{-L}^{L} \varphi(x) \mathrm{d} x+\int_{-L}^{L} v(t, x) \mathrm{d} x=\int_{-L}^{L} u_{0}(x)
$$

Thus, the choice $c_{0}=(2 L)^{-1} \int_{-L}^{L}\left(u_{0}(x)-\varphi(x)\right) \mathrm{d} x$ will ensure that $\int_{-L}^{L} v(t, x) \mathrm{d} x=$ 0 throughout the evolution. We get the following equation for $v$ (after a change of variable $x-c_{0} t \rightarrow x$ ),

$$
\mid \begin{aligned}
& v_{t}+\mathcal{L} v+y^{\prime}(t) \varphi^{\prime}(x+y(t))+v v^{\prime}=0 \\
& v(0, x)=u(0, x)-\varphi(x)-c_{0}=: f(x) \in H_{0}^{1}[-L, L]
\end{aligned}
$$

where $A$ is fixed in the definition of $y(t)$, say $A=\sup \{|\lambda|: \lambda<0, \lambda \in \sigma(\mathcal{L})\}+1$.

At this point of the argument, we have $\int_{-L}^{L} v(t, x) \mathrm{d} x=0$ and hence, the spectral projection of $v$ onto the eigen-direction $\psi=1$ stays trivially zero for all times $t$ (as if the eigen-direction 1 does not appear in the spectrum of $\mathcal{L}$ ). Thus, we can perform spectral projections as in (38) and continue the exact same argument from there on.

\section{REFERENCES}

[1] U. Frisch, Z.S. She, O. Thual,Viscoelastic behaviour of cellular solutions to the Kuramoto-Sivashinsky model. J. Fluid Mech. 168 (1986), 221240.

[2] I.G. Kevrekidis, B. Nicolaenko, J. Scovel, Back in the saddle again: a computer assisted study of the Kuramoto-Sivashinsky equation., SIAM J. Appl. Math. 50 (1990), no. 3, p. 760-790.

M. Stanislavova AND A. Stefanov Department of Mathematics University of Kansas 1460 Jayhawk Blvd Lawrence, KS 66045-7523, USA E-mail: stanis@math.ku.edu

A. Stefanov

E-mail: stefanov@math.ku.edu 